\title{
Authors' Reply to M.S. Raghuraman: “Intranasal Dexmedetomidine for Procedural Sedation in Children, a Suitable Alternative to Chloral Hydrate"
}

\author{
${\text { Giorgio } \operatorname{Cozzi}^{1} \text { (D) }}^{\text {Stefania Norbedo }}{ }^{1} \cdot$ Egidio Barbi $^{1}$
}

Published online: 28 June 2017

(c) Springer International Publishing AG 2017

We thank Dr./Professor Raghuraman [1] for pointing out the importance of considering also economical aspects related to the use of dexmedetomidine for procedural sedation in children. In accordance with the December 2016 Food and Drug Administration warning about sedatives in children [2], we think that dexmedetomidine will be more and more used in this setting. Therefore, we agree that future research should be focused on dexmedetomidine-related costs.

As we wrote in our manuscript [3], the bioavailability of dexmedetomidine via the oral route is poor in comparison with administration via the intravenous, intramuscular and intranasal routes [4]. Moreover, dexmedetomidine is odourless and tasteless and may be administered by nose without discomfort for the patient. Therefore, we think that the intranasal route is preferable to the oral route both for dexmedetomidine as premedication and as the main agent for sedation in children.

For what concerns oral and intranasal dexmedetomidine doses, we specified the dose ranges studied in children both for oral and intranasal administration of dexmedetomidine. The most studied doses of intranasal dexmedetomidine are 1 and $2 \mathrm{mcg} / \mathrm{kg}$ [5]. Nevertheless, several studies and, in particular, some comparing intranasal dexmedetomidine and oral chloral hydrate, also cited by Raghuraman,

This reply refers to the article available at doi:10.1007/s40272-0170245-1.

\section{Giorgio Cozzi}

giorgiocozzi@gmail.com

1 Emergency Department, Institute for Maternal and Child Health IRCCS "Burlo Garofolo", via dell'Istria 65/1, 34132 Trieste, Italy successfully used a dose of 3 and even $4 \mathrm{mcg} / \mathrm{kg}$, without clear evidence of an increase in adverse events related to these higher doses. Further studies focusing on higher doses of dexmedetomidine are needed to investigate if these may limit procedural safety.

Authors' Contributions All authors, NS, CG and BE, contributed equally to the manuscript. All authors, NS, CG and BE, read and approved the manuscript and its submission to the journal.

\section{Compliance with Ethical Standards}

Conflict of interest The authors NS, CG and BE declare that they do not have any conflict of interest.

Funding The authors, NS, CG and BE, declare that they did not receive any funding or grant for this manuscript.

\section{References}

1. Raghuraman MS. Comment on: "Intranasal dexmedetomidine for procedural sedation in children, a suitable alternative to chloral hydrate". Pediatr Drugs. 2017. doi:10.1007/s40272-017-0245-1.

2. U.S. Food and Drug Administration. Safety announcement: FDA review results in new warnings about using general anesthetics and sedation drugs in young children and pregnant women. 2016. http://www.fda.gov/downloads/Drugs/DrugSafety/UCM533197. pdf. Accessed 24 Jan 2017.

3. Cozzi G, Norbedo S, Barbi E. Intranasal dexmedetomidine forprocedural sedation in children, a suitable alternative to chloral hydrate. Pediatr Drugs. 2017. doi:10.2017/s40272-017-0217-5.

4. Mahmoud M, Mason KP. Dexmedetomidine: review, update, and future considerations of paediatrics perioperative and periprocedural applications and limitations. Br J Anaesth. 2015;115:171-82.

5. Rech MA, Barbas B, Chaney W, Greenhalgh E, Turck C. When to pick the nose: out-of-hospital and emergency department intranasal administration of medications. Ann Emerg Med. 2017. doi:10. 1016/j.annemergmed.2017.02.015. 